\title{
A parallel gradient method for the determination of the design point in probabilistic analysis \\ Y. Drobyshevski ${ }^{1} \quad$ H. Wadhwa ${ }^{2}$
}

(Received 19 December 2013; revised 8 January 2015)

\begin{abstract}
In the probabilistic analysis of engineering systems, the design point denotes a particular set of input parameters where the system response is most likely to take a given design value. It provides important information on the system behaviour and its sensitivity to input parameters. The design point is determined from the joint probability distribution function (PDF) of input parameters. Mathematically, the problem is equivalent to an isoperimetric problem: find a stationary point of the joint PDF subject to the given value of the system response. The proposed method depends on the response and the joint PDF being parallel at the stationary point. This requires the projection of the PDF gradient to be zero on the hyperplane orthogonal to the response
\end{abstract}

http://journal.austms.org.au/ojs/index.php/ANZIAMJ/article/view/7839 gives this article, (C) Austral. Mathematical Soc. 2015. Published January 23, 2015, as part of the Proceedings of the 11th Biennial Engineering Mathematics and Applications Conference. ISSN 1446-8735. (Print two pages per sheet of paper.) Copies of this article must not be made otherwise available on the internet; instead link directly to this URL for this article. 
gradient. Therefore, the design point is found by moving iteratively on the response surface in the direction of a non-zero projection vector until this vector vanishes. Convergence of this process is intuitively guaranteed. The method can be implemented for any number of input parameters. An example application is presented which demonstrates finding the most probable design metocean conditions for a floating structure. Such a problem is part of the response based analysis of offshore systems, which provided the initial motivation for this work.

\section{Contents}

1 Introduction

2 Problem formulation

C498

3 Parallel gradient method

C499

4 Heave motion of a spar

C503

5 Concluding remarks

C508

References

C511

\section{Introduction}

In the probabilistic analysis of engineering systems, design point is a set of input parameters where the system response is most likely to take a given design value. Within the structural reliability framework [1, 2], the input parameters are basic variables such as external conditions, forces and material properties, and the response may denote extreme motion, load or safety margins. Finding the design point (or several such points for a complex system) is an important part of probabilistic analysis. 
Finding the design point is not a new problem and several methods and proprietary programmes are available for solving such a problem. Most frequently, first order and second order reliability methods (FORM and SORM) are used, where the design point is obtained from a general reliability analysis. Such analysis involves a transformation of the basic variables to a set of normalized standard variables and approximating the response with a linear or quadratic function of the basic variables.

A similar problem is encountered in response based analysis (RBA) of offshore structures, which aims to predict extreme responses by analysing their statistics in site-specific metocean conditions. After these responses are determined, the inverse problem is solved: the most probable metocean conditions which cause extreme responses are determined. These metocean conditions are used to perform a more in-depth investigation of the system by detailed analysis and model testing. The description of the RBA methodology was discussed, for example, by Standing et al. [4] and Tromans et al. [5]. Finding the design points within RBA is simpler than the general reliability analysis formulation because both the design response and its probability are already known. This provides motivation for a simpler numerical method to be developed. Similar problems are also encountered in system operability assessments where combinations of the input parameters are sought under which the system is most likely to become inoperable.

This article presents a method for finding the design point for a given system response and probability density function (PDF) of input parameters. Section 2 formulates the problem, which is equivalent to an isoperimetric problem. In Section 3 the problem is solved using the parallel gradient condition of the response and PDF functions, which enables an efficient search process to be set up. A numerical example presented in Section 4 demonstrates finding the most probable design metocean condition for typical responses of a floating structure. In Section 5 features and applications of the method are discussed. 


\section{Problem formulation}

Consider a system described by the response $R(\vec{x})$, which is a probabilistic function of a random vector of $n$ input parameters:

$$
\vec{x}=\left(x_{1}, x_{2}, \ldots, x_{n}\right) .
$$

The vector components $x_{i}$ may be physical variables or their normalized values. The response distribution function is generally conditional on $\vec{x}$ :

$$
\mathrm{F}(\mathrm{R} \mid \vec{x})=\operatorname{Pr}(\mathrm{r}<\mathrm{R} \mid \vec{x}) .
$$

Randomness of the input parameters is described by the joint PDF $p(\vec{x})$. Therefore, the probability that the response exceeds a design value $R_{d}$ is

$$
q=\int_{\vec{x}} \int_{R>R_{d}}[1-F(R \mid \vec{x})] p(\vec{x}) d R d \vec{x} .
$$

The integration is performed over the complete $\vec{x}$-space and over the range where the response exceeds the design value. For a given $q$ the corresponding design value $R_{d}$ is found from equation (3); this is a typical reliability analysis formulation.

In the following it is assumed that the design response $R_{d}$ and the associated exceedance probability $\mathrm{q}$ are already determined, such that the response is considered as a deterministic function. By formally using the probability distribution

$$
F(R)= \begin{cases}1 & \text { for } R<R_{d}, \\ 0 & \text { for } R \geqslant R_{d}\end{cases}
$$

one re-writes equation (3) as

$$
q=\int_{g(\vec{x})<0} p(\vec{x}) d \vec{x} .
$$


The integration is now performed over the 'failure set' defined by the failure function

$$
g(\vec{x})=R_{d}-R(\vec{x}) .
$$

The failure function (6) defines the deterministic 'failure surface' in $(n+$ 1 ) dimensions and separates 'safe' and 'failure' conditions. The $\vec{x}$ values where $g(\vec{x})=0$ form the trace of the failure surface in the $\vec{x}$-space, referred to as the response surface. The problem is to determine a particular design point $\vec{x}_{\mathrm{d}}$ on the response surface which provides the maximum contribution to the probability integral (5). Therefore, the design point must be the point of a conditional extremum of the joint PDF:

$$
g\left(\vec{x}_{d}\right)=0, \quad p\left(\vec{x}_{d}\right)=\max \{p(\vec{x}) \mid g(\vec{x})=0\} .
$$

The design point $\vec{x}_{d}$ defines the most probable combination of input parameters for which system response $R_{d}$ will occur.

\section{Parallel gradient method}

The problem (7) is interpreted as an isoperimetric problem, which is solved by the method of Lagrange multipliers $[6,7]$. One seeks the stationary point of

$$
f(\vec{x}, \lambda)=p(\vec{x})-\lambda R(\vec{x}),
$$

where $\lambda$ is unknown. Therefore, the design point is found by requiring

$$
R(\vec{x})=R_{d}, \quad \frac{\partial p(\vec{x})}{\partial x_{i}}-\lambda \frac{\partial R(\vec{x})}{\partial x_{i}}=0, \quad i=1,2, \ldots, n .
$$

Therefore, the problem is reduced to a system of $(n+1)$ equations in the $\vec{x}$-domain. In many applications the response surface $R(\vec{x})=R_{d}$ and PDF function $p(\vec{x})$ can only be determined numerically by specialized software. Therefore, an efficient method is required for solving system (9). 


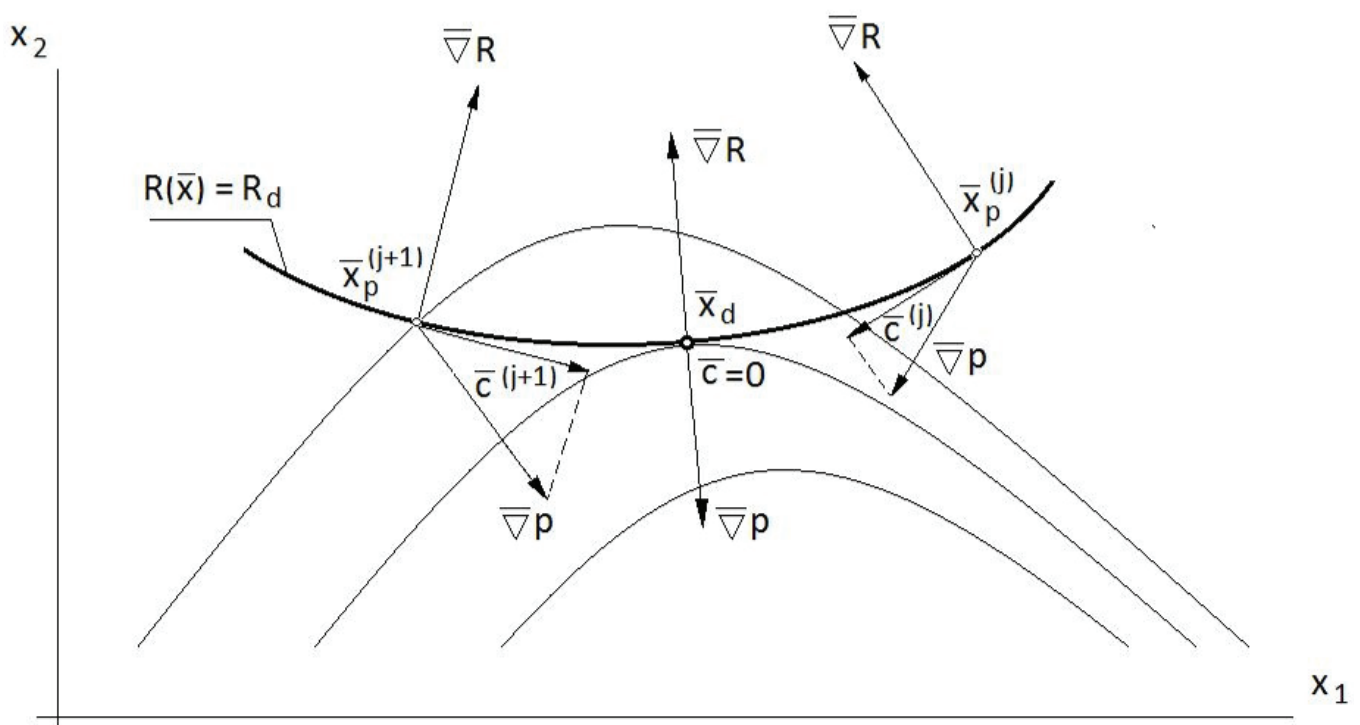

Figure 1: Parallel gradient condition at the design point.

The proposed method is based on a property which follows immediately from (9): at the stationary point $\vec{x}_{d}$ the gradients of the response function $\vec{\nabla} R(\vec{x})$ and PDF $\vec{\nabla} p(\vec{x})$ must be parallel. The requirement of parallel gradients is met if and only if the projection $\vec{c}$ of the gradient $\vec{\nabla} p(\vec{x})$ on the hyper-plane tangential to the response surface is zero (Figure 1). Otherwise, for any point $\vec{x}_{p}$ on the response surface the nonzero vector $\vec{c}$ indicates the direction where the design point $\vec{x}_{d}$ should be sought. This property enables the search process to be set up by moving the point $\vec{x}_{p}$ iteratively in the direction of the projection vector $\overrightarrow{\mathbf{c}}$ until this projection vector vanishes. The iteration process is described by the following steps.

First, for a given point $\vec{x}_{p}$ on the response surface the response gradient and components of its unit vector $\vec{a}$ are calculated:

$$
a_{i}\left(\vec{x}_{p}\right)=\frac{1}{\left|\vec{\nabla} R\left(\vec{x}_{p}\right)\right|} \frac{\partial R}{\partial x_{i}}\left(\vec{x}_{p}\right), \quad i=1,2, \ldots, n
$$


Then, the PDF gradient $\vec{\nabla} \mathrm{p}(\vec{x})$ and its projection $\vec{c}=\left(c_{k}\right)_{k=1}^{n}$ on the tangential hyper-plane are calculated:

$$
c_{k}=\frac{\partial p}{\partial x_{k}}\left(\vec{x}_{p}\right)-a_{k} \sum_{i=1}^{n} a_{i} \frac{\partial p}{\partial x_{i}}\left(\vec{x}_{p}\right), \quad k=1,2, \ldots, n .
$$

Finally, the next iteration step moves the current point $\vec{x}_{p}=\vec{x}_{p}^{(j)}$ on the response surface towards the next point

$$
\vec{x}_{0}^{(j+1)}=\vec{x}_{p}^{(j)}+\frac{\vec{c}^{(j)}}{\left|\vec{\nabla} p\left(\vec{x}_{p}^{(j)}\right)\right|} d^{(j)}, \quad j=1,2, \ldots
$$

Here, $d^{(j)}$ is a scalar parameter which controls the magnitude of the $j$ th iteration step, and $\vec{x}_{0}^{(j+1)}$ is the initial point of the next iteration. The value of the control parameter is estimated by making a trial step with a trial value $d_{T}^{(j)}$ and using the result for the trial vector $\vec{c}_{T}^{(j+1)}$ to obtain the final control parameter value

$$
d^{(j)}=d_{T}^{(j)} \frac{c_{k}^{(j)}}{c_{k}^{(j)}-c_{T k}^{(j+1)}}, \quad k=\operatorname{argmax}_{i=1, \ldots, n}\left|c_{i}^{(j)}\right| .
$$

In the above equation the control parameter $d^{(j)}$ is determined by linear interpolation between the current and the trial points in order to minimize the governing $k$-component of the projection vector $\vec{c}^{(j)}$. The $k$-component of the trial vector $c_{T}^{(j+1)}$ is $c_{T k}^{(j+1)}$. Other methods for optimizing the control parameter may be possible, which should be investigated further.

The iteration process continues until the projection vector $\vec{c}$ becomes small compared with the PDF gradient. The relative error measures the extent to which the two gradients are parallel:

$$
\epsilon=\frac{\|\overrightarrow{\mathbf{c}}\|}{\left\|\vec{\nabla} p\left(\vec{x}_{p}\right)\right\|} .
$$

The relative error is equivalent to the tangent of the angle between the two gradient vectors and should vanish at the design point. 
The search process must ensure that the response surface is accurately followed. Because the initial point for the $(j+1)$ th iteration $\vec{x}_{0}^{(j+1)}$ defined by (12) may deviate from the response surface it should be checked by calculating the response $R\left(\vec{x}_{0}^{(j+1)}\right)=R_{0}$. If the difference $\left|R_{d}-R_{0}\right|$ is large, then the correct point $\vec{x}_{p}^{(j+1)}$ is found by using the hyper-plane approximation for the response surface (superscript $(j+1)$ is omitted for brevity)

$$
\mathrm{R}(\overrightarrow{\mathrm{x}})=\mathrm{R}_{0}+\left|\vec{\nabla} \mathrm{R}\left(\vec{x}_{0}\right)\right| \sum_{i=1}^{n} \mathrm{a}_{i 0}\left(x_{i}-x_{0}\right) .
$$

Here $a_{i 0}$ for $i=1, \ldots, n$ are components of the unit gradient vector $\vec{a}_{0}$ of the response at the initial point. Assuming that the hyper-plane (15) passes through the response surface, the projection of the initial point $\vec{x}_{0}$ on this surface is

$$
\vec{x}_{p h}=\vec{x}_{0}+\left(R_{d}-R_{0}\right) \vec{a}_{0} .
$$

The response $R\left(\vec{x}_{\text {ph }}\right)$ is now calculated and if the difference $\left|R_{d}-R\left(\vec{x}_{p h}\right)\right|$ is still large, then the hyper-plane approximation should be repeated. Alternatively, the point (16) and the response gradient at the initial point are assumed to be applicable to the response surface:

$$
\vec{x}_{p}^{(j+1)}=\vec{x}_{p h}, \quad \vec{a}\left(\vec{x}_{p}\right)=\overrightarrow{a_{0}}\left(\vec{x}_{0}\right) .
$$

The $(j+1)$ th iteration is now performed by applying equation (12) to obtain the next initial point $\vec{x}_{0}^{(j+2)}$. Each hyper-plane approximation requires $(2 n+1)$ response calculations, and if iteration steps $\mathrm{d}^{(\mathfrak{j})}$ are not large and the response surface is smooth, then one hyper-plane approximation per iteration is usually sufficient. This enables the same response calculations to be used for both the hyper-plane approximation (15) and for the projection vector (11), which makes the iteration process reasonably efficient. In other cases, several hyperplane iterations may be required to accurately follow the response surface.

Convergence of the iteration process to the design point is intuitively guaranteed. The method of hyper-plane approximations enables the search process to be started from any initial point $\vec{x}_{0}$ for any target response $R_{d}$. Depending 
on the system type, response properties and nature of the input parameters (for example, peaks and valleys in the PDF $p(\vec{x})$ ) several design points may exist for the same response with different values $p\left(\vec{x}_{d}\right)$. The validity and relative importance of such design points require engineering assessment. To identify all design points, the analysis may need to be started from different initial conditions to converge to the design point in the expected region.

\section{Heave motion of a spar}

This example is intended to illustrate the method on a simple system for which the results are easily interpreted. The proposed method is used to find the most probable design metocean conditions for heave response of a simple floating structure, a vertical cylinder with large draft (spar). Under the assumptions of linear wave theory and motions, heave motion of the spar $\zeta(t)$ is described by the differential equation

$$
(\mathrm{m}+\mu) \ddot{\zeta}+\lambda \dot{\zeta}+\rho g A \zeta=\mathrm{F}(\mathrm{t}) .
$$

Here $m$ is the mass of the structure, $A$ is the water plane area, $\mu$ and $\lambda$ are the hydrodynamic added mass and damping coefficient, respectively, $F(t)$ is the wave exciting force, and $\rho=1025 \mathrm{~kg} \mathrm{~m}^{-3}$ and $\mathrm{g}=9.81 \mathrm{~m} \mathrm{~s}^{-2}$ denote the sea water density and the acceleration of gravity, respectively.

Using the elevation of incident waves of amplitude $r$ and frequency $\sigma$ in the complex form $\zeta_{w}(t)=r e^{i \sigma t}$ and simplifying the diffraction effects, one obtains an approximate solution for the heave transfer function

$$
\zeta(\mathrm{t})=\zeta_{A}(\sigma) e^{i \sigma t} \quad \text { where } \quad \zeta_{A}(\sigma)=\mathrm{r} \frac{\left(\omega^{2}-f \sigma^{2}\right)+2 i v \sigma}{\left(\omega^{2}-\sigma^{2}\right)+2 i v \sigma} .
$$

Here the natural frequency $\omega$, damping coefficient $v$ and inertia parameter $f$ are defined by

$$
\omega=\sqrt{\frac{\rho g A}{m+\mu}}, \quad v=\frac{\lambda}{2(m+\mu)}, \quad f=\frac{\mu}{m+\mu} .
$$




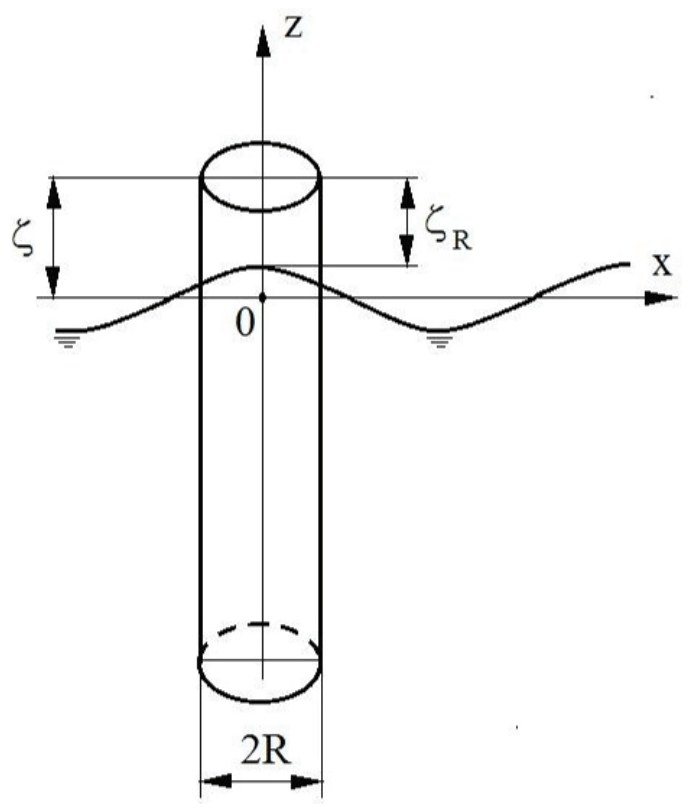

Figure 2: Definition of heave motion of the spar.

Equations (18)-(20) describe a linear formulation for the spar heave motion which is discussed, for example, by Morgan [9]. Two responses will be considered in this example: heave (absolute vertical motion) and relative vertical motion between the wave profile and the spar (Figure 2). By using (19) one expresses these responses in terms of the response amplitude operators (RAO):

$$
\begin{aligned}
\zeta(\sigma) & =\frac{\left|\zeta_{A}\right|}{r}=\sqrt{\frac{\left(\omega^{2}-f \sigma^{2}\right)^{2}+(2 v \sigma)^{2}}{\left(\omega^{2}-\sigma^{2}\right)+(2 v \sigma)^{2}}}, \\
\zeta_{R} A(\sigma) & =\frac{\left|\zeta_{R A}\right|}{r}=\frac{\sigma^{2}(1-f)}{\sqrt{\left(\omega^{2}-\sigma^{2}\right)+(2 v \sigma)^{2}}} .
\end{aligned}
$$

Table 1 presents particulars of the spar, which are close to those of some real structures of this type; RAO (21) are plotted in Figure 3. Both RAO display 
Table 1: Particulars and hydrodynamic properties of spar.

\begin{tabular}{llll} 
Description & Notation & Unit & Value \\
\hline water plane radius & $\mathrm{R}$ & $\mathrm{m}$ & 10.97 \\
draft & $\mathrm{D}$ & $\mathrm{m}$ & 198.12 \\
freeboard & $\mathrm{F}$ & $\mathrm{m}$ & 16.76 \\
damping ratio & $\lambda / \lambda_{\text {crit }}$ & - & 0.05 \\
water plane area & $\mathrm{A}$ & $\mathrm{m}^{2}$ & 378.3 \\
mass including trapped water & $\mathrm{m}$ & $\mathrm{kg}$ & $7.68 .5 \times 10^{7}$ \\
added mass & $\mu$ & $\mathrm{kg}$ & $8.5 \times 10^{6}$ \\
damping coefficient critical & $\lambda_{\text {crit }}$ & $\mathrm{N} \mathrm{m} \mathrm{s}^{-1}$ & $3.6 \times 10^{7}$ \\
damping coefficient actual & $\lambda$ & $\mathrm{N} \mathrm{m} \mathrm{s}^{-1}$ & $1.8 \times 10^{6}$ \\
natural heave frequency & $\omega$ & $\mathrm{rad} \mathrm{s}^{-1}$ & 0.21 \\
natural heave period & $\tau$ & $\mathrm{s}$ & 29.8 \\
damping ratio & $v$ & - & 0.011 \\
inertia parameter & $\mathrm{f}$ & - & 0.10
\end{tabular}

resonant behaviour in the vicinity of the natural heave frequency but their behaviour outside the resonant zone is different: heave motion tends to zero at high frequencies as short waves do not produce sufficient excitation; while the relative motion becomes equal to the wave amplitude. These tendencies are reversed at small frequencies.

The most probable maximum response of the structure over the three hour duration $z_{\text {MPM }}$ due to an irregular sea state with the significant wave height $\mathrm{H}_{\mathrm{s}}$ and peak period $T_{p}$ is

$$
z_{\mathrm{MPM}}=\sqrt{2 M_{0} \log \left(\frac{3 \times 60 \times 60}{\tau_{z}}\right)} \quad \text { with } \quad \tau_{z}=2 \pi \sqrt{\frac{M_{0}}{M_{2}}} .
$$

Here $\tau_{z}$ is the zero-up-crossing period, and $M_{0}$ and $M_{2}$ are two spectral moments. Equation (22) is based on the assumption that each three hour irregular sea state is a stationary Gaussian process described by the wave 


\section{$\zeta,[\mathrm{m} / \mathrm{m}]$}

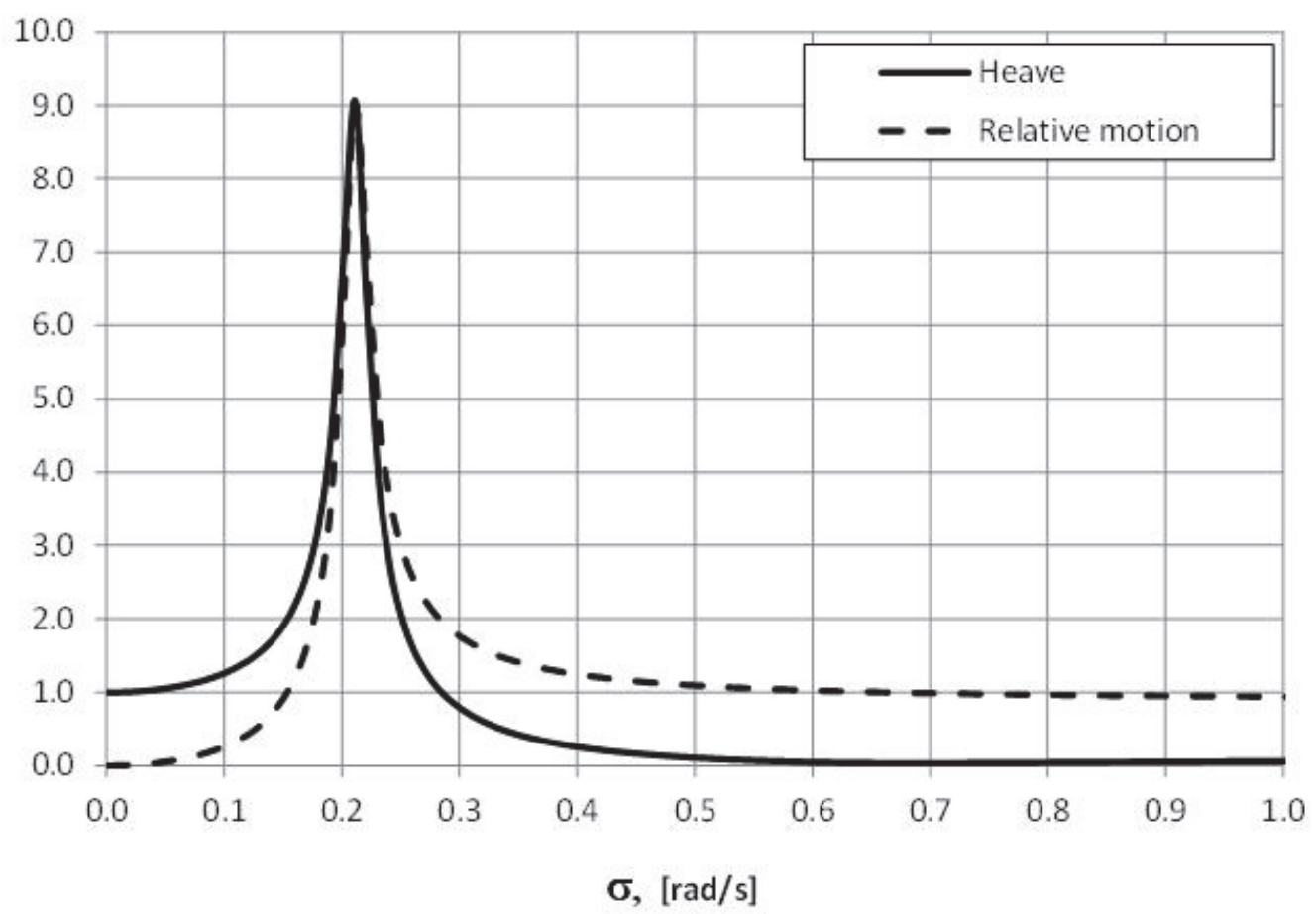

Figure 3: RAO of heave and relative motion of the spar.

energy spectrum $S(\sigma)$ and the response amplitudes follow Rayleigh distributions. Equations (21)-(22) define the response functions $z_{\text {MPM }}=f\left(H_{s}, T_{p}\right)$ for the two responses of interest.

In this example responses depend only on the significant wave height $\mathrm{H}_{s}$ and peak period $\mathrm{T}_{p}$; hence a joint metocean PDF of these two variables is used. Bitner-Gregersen [8] developed an analytical formulation for the joint PDF of $\mathrm{H}_{s}$ and $\mathrm{T}_{\mathrm{p}}$ in the North Sea which utilized a Weibull marginal distribution for $\mathrm{H}_{s}$ and conditional log-normal distribution for $T_{p}$. In this formulation the long-crested sea states are described by a JONSWAP spectrum [9] and the 
Table 2: Initial and most probable design metocean conditions for the 100-year sea state with $H_{a}$ and $R$ in units of metres and $T_{p}$ in seconds.

\begin{tabular}{l|ccc|ccc|cc} 
& \multicolumn{3}{|c|}{ Initial point } & \multicolumn{3}{c|}{ Design point } & \multicolumn{2}{c}{ Sensitivity } \\
Response & $\mathrm{H}_{s}$ & $\mathrm{~T}_{\mathrm{p}}$ & $\mathrm{R}$ & $\mathrm{H}_{s}$ & $\mathrm{~T}_{\mathrm{p}}$ & $\mathrm{R}$ & $\mathrm{a}_{1}$ & $\mathrm{a}_{2}$ \\
\hline heave motion & 16.91 & 17.44 & 6.75 & 15.16 & 17.82 & 6.75 & 0.22 & 0.97 \\
relative motion & 16.91 & 17.44 & 20.59 & 16.43 & 17.85 & 20.59 & 0.63 & 0.78
\end{tabular}

maximum 100-year return period sea state is $H_{s}=16.91 \mathrm{~m}$ and $T_{p}=17.44 \mathrm{~s}$. Example 1 (Most probable conditions for heave and relative motion). The maximum amplitudes of the heave and relative motion were calculated for the maximum 100-year sea state by applying equation (22). Then the most probable conditions under which the same responses may occur were determined. Results are presented in Table 2, which also shows the sensitivity factors - components of the unit vector $\vec{a}$ of the response gradient at the design point.

Figure 4 demonstrates the iteration process for the heave response. The responses calculated for the maximum 100-year sea state are more likely to occur in different conditions. The heave motion of $6.75 \mathrm{~m}$ in particular will most probably be generated by a less severe sea state with $\mathrm{H}_{s}=15.16 \mathrm{~m}$, which will occur more frequently than once in 100 years. This indicates that the selected 100-year sea state is not the worst with respect to heave, and other conditions should be examined. This can be done by calculating the response at several points over the 100-year IFORM contour [3], or by conducting a comprehensive response based analysis. Sensitivity factors indicate that the heave is more sensitive to the peak period whereas the relative motion is sensitive to both the wave height and the period.

Example 2 (Most probable condition for zero air-gap). Because the relative motion of $20.59 \mathrm{~m}$ predicted in Example 1 exceeds the static freeboard of the spar $(16.76 \mathrm{~m})$, the topside deck is likely to be exposed to wave loading. It is 
Table 3: Initial and most probable design metocean conditions for zero air-gap with $\mathrm{H}_{s}$ and $\mathrm{R}$ in units of metres and $\mathrm{T}_{\mathrm{p}}$ in seconds.

\begin{tabular}{l|ccc|ccc|cc} 
& \multicolumn{3}{|c|}{ Initial point } & \multicolumn{3}{c|}{ Design point } & \multicolumn{2}{|c}{ Sensitivity } \\
Response & $\mathrm{H}_{s}$ & $\mathrm{~T}_{\mathrm{p}}$ & $\mathrm{R}$ & $\mathrm{H}_{s}$ & $\mathrm{~T}_{\mathrm{p}}$ & $\mathrm{R}$ & $\mathrm{a}_{1}$ & $\mathrm{a}_{2}$ \\
\hline relative motion & 16.91 & 17.44 & 20.59 & 14.35 & 16.78 & 16.76 & 0.77 & 0.64
\end{tabular}

useful to determine the most probable condition corresponding to the dynamic air-gap being zero, that is, when the relative motion is exactly $16.76 \mathrm{~m}$. Results of this analysis are presented in Table 3 and in Figure 5.

The most probable condition for the zero air-gap is found to be well below the 100-year isoline; it is in this condition the spar is most likely to experience first wave loading on its topside. If the spar is intended to operate in harsher environments, then following the same process one can determine a range of negative air-gap conditions. These conditions can then be investigated to quantify the wave loads and to assess the structure behaviour.

\section{Concluding remarks}

The proposed parallel gradient method for finding the design point is not limited to a specific response and can be implemented for any number of $\vec{x}$ dimensions. The vector $\vec{x}$ may describe any parameters, including variability in the response itself or variability in the system capacity.

Compared with FORM and SORM the proposed method is less general, as it solves a narrower task. On the other hand, it does not involve any simplifying assumptions for the response surface and avoids transformation of the $\vec{x}$ space into normalized standard variables. Undertaking the search within the space of physical variables has the benefit of ease of interpretation of the results. 


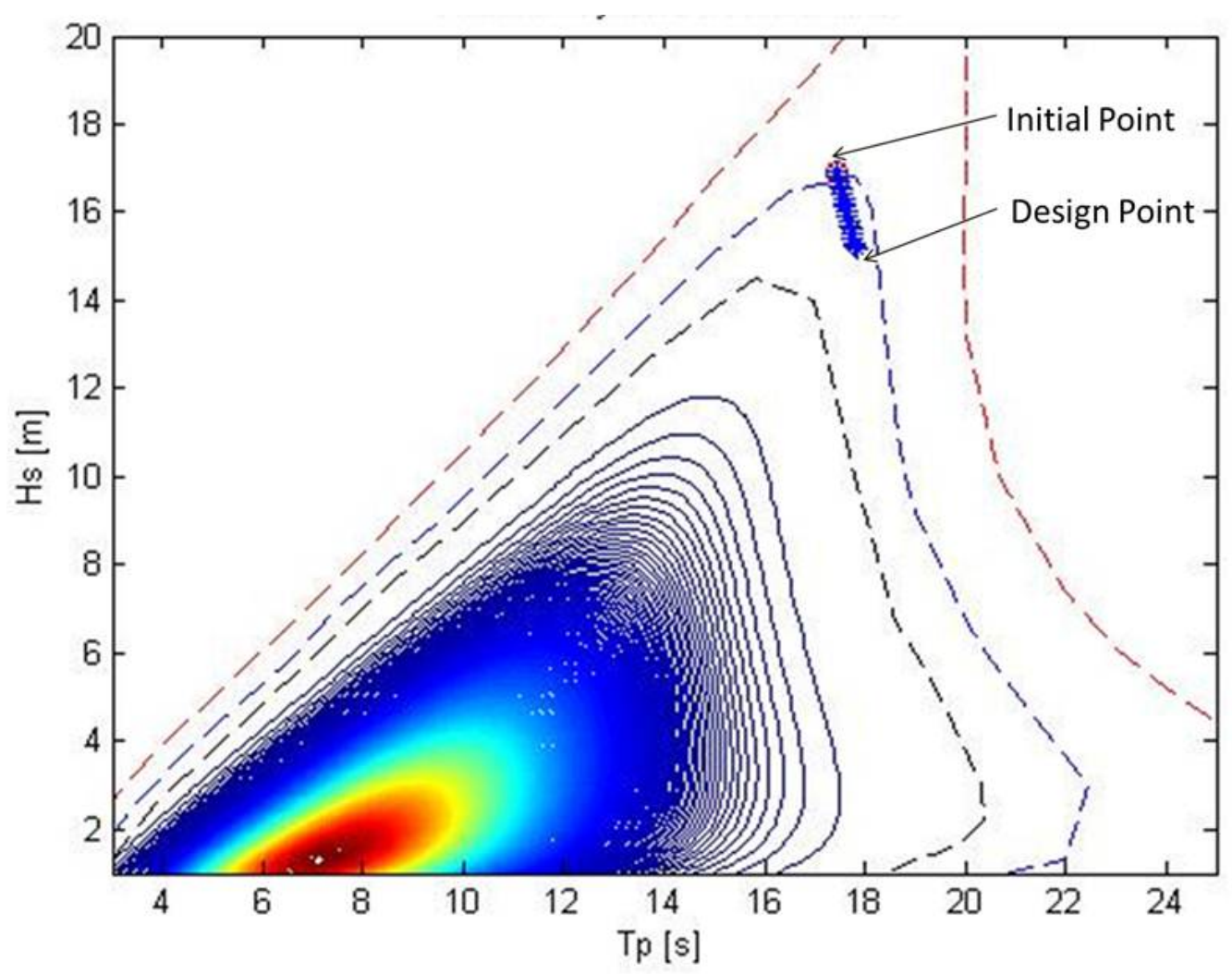

Figure 4: Most probable design metocean condition for the heave of $6.75 \mathrm{~m}$.

The method can be used as a starting point for a more general reliability analysis. The design point and the sensitivity factors define the hyper-plane in the $\vec{x}$ space, which approximates the response surface within the FORM framework. These parameters enable the probability integral in (3) (or (5)) to be calculated more accurately by using, for example, a Monte-Carlo technique with importance-sampling Winterstein [2]. 


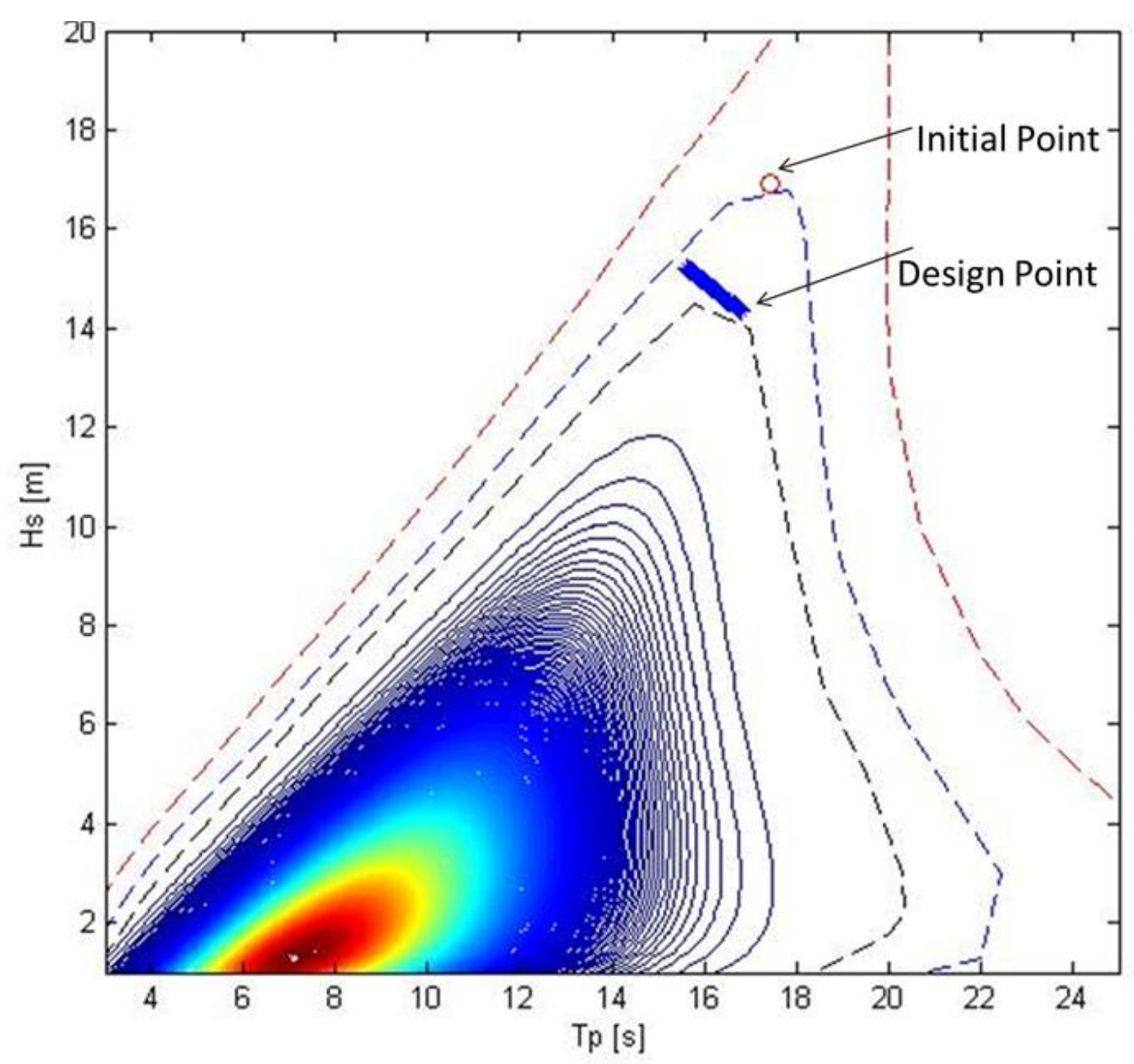

Figure 5: Most probable design metocean condition for relative motion of $16.76 \mathrm{~m}$. 
Acknowledgements We are grateful to INTESEA Pty Ltd for support and permission to publish this work. The authors also thank Dr James Whelan for useful discussions.

\section{References}

[1] H. O. Madsen, S. Krenk and N. C. Lind. Methods of structural safety. Dover, 1986.

http://store.doverpublications.com/0486445976.html. C496

[2] S. R. Winterstein. An introduction to structural reliability, FORM, and LRFD design. Technical report, 2011.

https://dl.dropboxusercontent.com/u/3121325/lrfd/lrfd.pdf. C496, C509

[3] S. Winterstein, T. C. Ude, C. A. Cornell, P. Bjerager and S. Haver. Environmental parameters for extreme response: inverse FORM with omission sensitivity. Structural Safety and Reliability, Proc., ICOSSAR '93, A. A. Balkema, 1994. C507

[4] R. G. Standing, R. Eichaker, H. D. Lawes, B. Campbell and R. B. Corr. Benefits of applying response based design methods to deepwater FPSOs. Offshore Tech. Conf., 2002. doi:10.4043/14232-MS. C497

[5] P. S. Tromans and L. Vanderschuren. Response based design conditions in the North Sea: application of a new method. Offshore Tech. Conf., 1995. doi:10.4043/7683-MS. C497

[6] I. M. Gefland and S. V. Fomin. Calculus of variations. Dover, 2000. http://store.doverpublications.com/0486414485.html. C499

[7] J. Mathews and R. L. Walkers. Mathematical Methods of Physics. W. A. Benjamin, 1964. C499 
[8] E. M. Bitner-Gregersen. Joint probabilistic description of combined seas. ASME Proc., Safety and Reliability, OMAE2005-67382:169-180, 2005. doi:10.1115/OMAE2005-67382. C506

[9] N. Morgan. Marine technology reference book. Butterworth-Heinemann, 1990. C504, C506

\section{Author addresses}

1. Y. Drobyshevski, intecsea Pty Ltd and Australian Maritime College (University of Tasmania), Australia. mailto:yuriy.drobyshevski@intecsea.com

2. H. Wadhwa, Intecsea Pty Ltd and School of Mechanical Engineering, The University of Western Australia, Australia. mailto:hema.wadhwa@intecsea.com 